\title{
A new benchmark method for the identification and assessment of alternative technologies
}

\author{
D. Russo', T. Montecchí, S. Duci \\ ${ }^{1}$ University of Bergamo, viale Marconi 5, Dalmine - Italy, \\ ${ }^{2}$ Bigflo srl, via Galvani 2E, Dalmine - Italy \\ davide.russo@unibg.it
}

\begin{abstract}
Every time we need to develop a new technical system or improve an existing one, the evaluation and the identification of potential alternative technologies assume a central role. In conventional decision making strategies, the decision to invest in one or more alternative technologies is performed by weighting benefits and risks of each alternative. Both the identification and weighting of alternative technologies are usually performed without a systematic methodology, relying on the experts' knowledge and somehow on the unquestionable judgment of leaders. The main risks of these approaches are the strong subjectivity of the evaluation and the strong dependence of alternative technologies from experts' knowledge. In the worst case scenario, this situation may lead to an ineffective investment. The proposed methodology, called "KOMpetitive intelligence", is based on a systematic approach, combining Knowledge Search and Evolutionary Problem Solving developed through several years of experimentation and specifically built to overcome the aforementioned problems. First, knowledge of experts is integrated with knowledge extracted from patents, market analysis, scientific literature and commercial literature. Second, the generation of new alternative technologies is supported with a systematic theory of problem solving and knowledge transfer. Third, decision making and the definition of an innovation strategy are supported with a concise diagram that summarizes the gathered knowledge and facilitates the assessment of the alternative technologies. As a result, gathered knowledge and problem solving foster the ability of experts to identify and assess alternative technologies. In some cases, completely new technologies are identified to be suitable with the considered application. The graphical summary allows experts and leaders to have a comprehensive and fast overview on the situation, increasing awareness and consistency of decision making.
\end{abstract}

Key words: benchmark, competitive intelligence, technology transfer, TRIZ, problem solving, patent

\section{Introduction}

The present work is part of a broader methodology of problem solving, called Spark. Spark has been developed at the University of Bergamo and part of it has been implemented in a patented software [1]. It consists of 5 steps to innovate a product or a technology and is mainly based on TRIZ, the Theory of Inventive Problem Solving created by Altshuller [2]. In the practice of innovation, TRIZ is a well-known theory; its founder sustains that the ability to invent something is not just due to personal talent and capabilities. Everyone can be more creative by combining its talent and tacit knowledge with proper tools. Specifically, Altshuller extracted all possible paths that lead to an invention from a wide pool of patents. The generalization of these paths became the fundamentals of TRIZ, which were concretized in several tools, such as the 40 Inventive Principles, the 76 standard solutions [3] and the Laws of technical system evolution [4]. However, TRIZ alone does not cover all the aspects of innovation. With Spark, TRIZ has been integrated with other methodologies, especially to include marketing aspects and to foster the problem solving activities.

The first two steps of Spark were originally developed to support the correct formulation of the problem in order to be sure we do not look for good solutions to the wrong problem. Both if we need to eliminate a harmful effect and improve an existing function, introducing a radically new one chosen from different technologies, we must be sure in advance that the new solution really improves the right requirements. The highest risk of a problem 
solving based on trial and error is to solve a problem creating a new one even worst, or improving the product with something that the customer does not perceive as a real benefit. A correct formulation of the initial problem is necessary to identify the set of requirements with the highest priority. The steps of problem formulation have been further developed and improved, and nowadays, they are proposed as an independent systematic methodology called "KOMpetitive intelligence".

For this work, our extensive methodology aims to help engineers, decision makers and managers in choosing the best technology at the state of the art and even improving an existing one transferring ideas from other fields and identifying the best directions/requirements to innovate. An overview of this methodology is proposed in the next session, together with an exemplary case study showing how very different technologies can be compared with a quantitative and objective approach. For the sake of brevity, the following three steps of the Spark process, conceived for improving the most critical requirement of the chosen technology are not presented in this article.

\section{Competitive Intelligence methodology}

The competitive intelligence methodology consists of two phases: (1) information gathering and (2) requirements evaluation.

\section{1) Information gathering.}

The goal of this phase is to collect quantitative data for making technology/product positioning.

First, we list all the requirements of the technology (i.e. for example for air quality sensors are the sensitivity, selectivity, calibration, response time, etc. ). A specific problem solving phase is dedicated to identify new desired functions/requirements.

Then we analyze each requirement separately providing:

- a knowledge search based on three documentary sources: patent, scientific literature and web. These knowledge searches aim to position the product analyzing the best solutions at the state of the art. In particular, from patents we extract the state of the art of alternative technologies, the technological positioning among competitors, technological patent trends and peculiar solutions. From scientific publications, we identify extra patent activities and collaborations between academia and enterprise. From the web and catalogs, we understand how the product is positioned among direct competitors and how requirements are communicated to the users and we verify if solutions disclosed in patents or publications are already on the market or still at the research level.

- Problem solving is performed in order to gain an evolutionary perspective on the existing and future technologies. From a methodological point of view we adopted the evolutionary tools from TRIZ as i.e. the laws of technical system evolution, and 76 standards solutions for understanding how existing technologies can evolve. Regarding knowledge searches, we used KOMPAT $^{\circledR}[5,6]$, a semantic knowledge search engine developed by BiGFLO [www.bigflo.it], allowing an automatic identification of patented alternative technologies based on different physical effects.

\section{2) Requirements evaluation.}

The goal of this phase is to weight all requirements in terms of market potential combining Design, Manufacturing, Quality and Marketing points of view.

Experts from all different areas evaluate each requirement according to two parameters: importance and satisfaction. Importance is reflected in goal-oriented search attributes that consumers actively look for in the target product and consider when making a purchase decision [7]. In other words, it is the degree to influence the customer decision. All past company investments are taken into account. Satisfaction is a measure of how products and services supplied by a company meet or surpass customer expectation [8].

Using two evaluation parameters allow a safer decision. Here an example about a mobile phone is given. If we consider what customer thinks about the battery life and the display definition, both requirements are very important. However, if we ask about their satisfaction, a big difference appears: existent display definition fully satisfies them while battery life could be much more long-lasting (one week against the actual one-day life).

In our method, importance is assessed combining quantitative analysis based on patent intelligence and screening of competitor brochures, with R\&D and Marketing audits. Audits are influenced by a specific Design activity (based on TRIZ), conceived for suggesting future evolutionary/technological scenarios. Satisfaction is assessed only by marketing staff. Importance and satisfaction help to calculate the market potential of the 
benefit as a decisive factor of customer value. Requirements are ranked by market potential index that is calculated with a formula derived from Ulwick [9]:

$$
M P=I+(I-S)
$$

Where, $M P$ is the market potential index, $I$ is the importance and $S$ is the satisfaction.

Finally, importance and satisfaction are displayed on a Cartesian graph taking into account the average of all experts' judgments (see fig. 1).

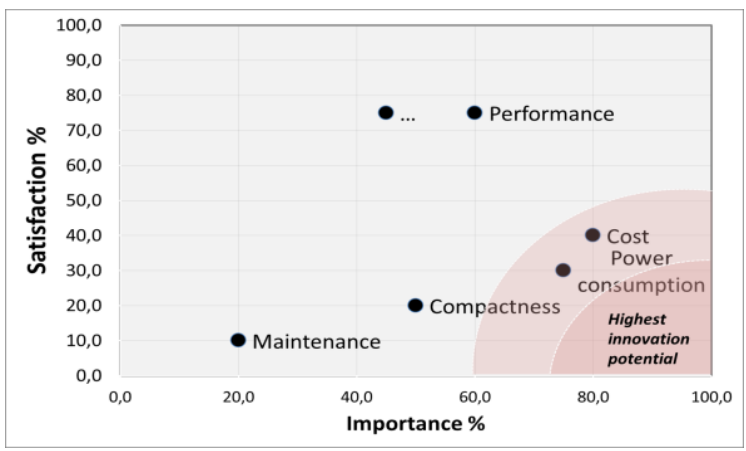

Fig. 1. Requirements ranking mapped on the Importance-Satisfaction diagram.

This diagram is a powerful tool for fixing the innovation product strategy. It allows to take awareness on which requirements have the highest market potential. They are clearly visualized in the right bottom corner of the graph and they represent the requirements with high importance and low satisfaction. Working on these requirements generates high value for the customer.

We adopt this rank for quantitatively comparing alternative technologies. The winner technology will be the technology with the highest average satisfaction of all requirements.

\section{Case study}

In this exemplary case study, the authors considered sensors for monitoring the level of pressure in a gas circuit breaker.

First, we list all the requirements of the sensor: cost, maintenance, compactness, performance, sensitivity, calibration, selectivity, response time, data transmission, data security, precision, power consumption.

Using KOMPAT software we identify a list of potential physical effects/technologies that can be used to measure the pressure, as shown in figure 2. This output is then converted into research targets for planning the info gathering phase in patent, non-patent literature and brochures. Figure 2 shows a partial list of alternative technologies for measuring pressure inside gas insulated circuit breakers. In yellow nine technologies already present into the gas breakers field, and in green three potential new physical effects to be transferred from other fields.

\section{1) Information gathering.}

The aim of this phase is to deeply analyze all requirements (by combining knowledge search and problem solving activity), collecting all information useful to compare technologies looking at a single requirement at time

For example in the "performance" requirement analysis, we identified over than 400 patents. Figure 3 shows the acceleration in the last 5 years of electrical and vibrating devices. Time distribution of main players allows to understand who is still working in very recent years (e.g. Hitachi and Siemens), and who has peak activity only in the past (Meidensha, Toshiba and GE).

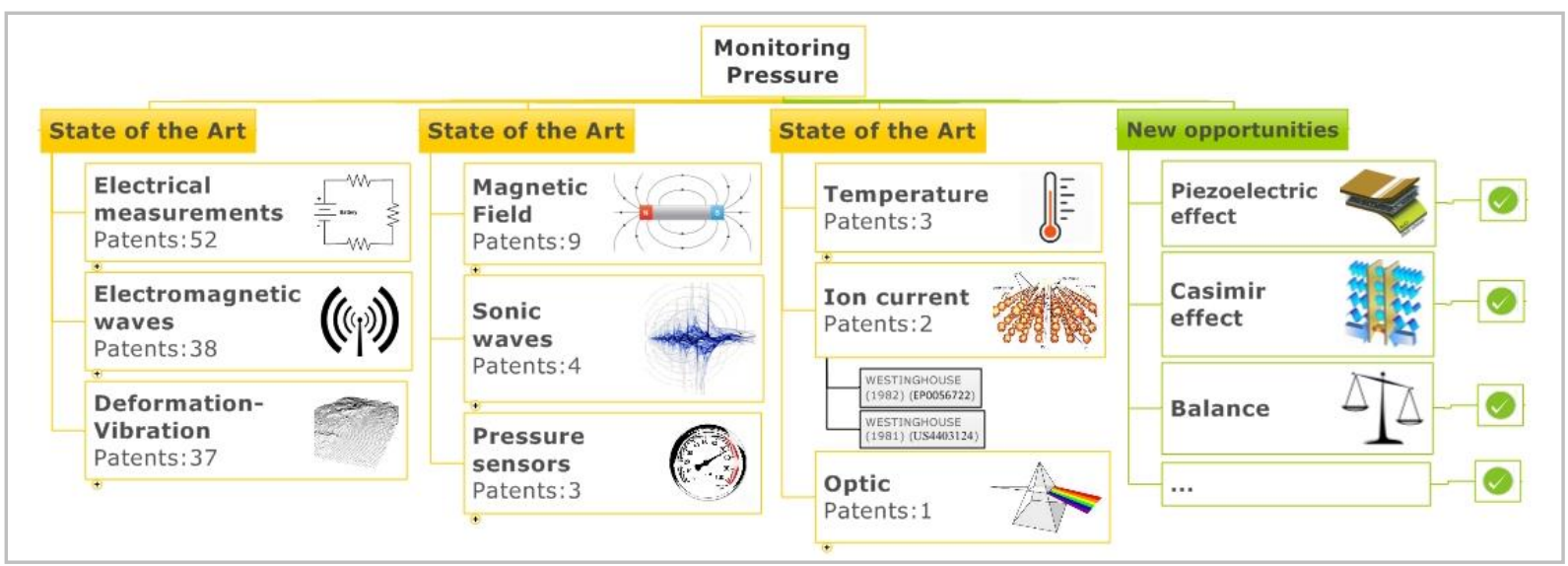

Fig. 2. Pressure sensors - State of the art and future technologies. 


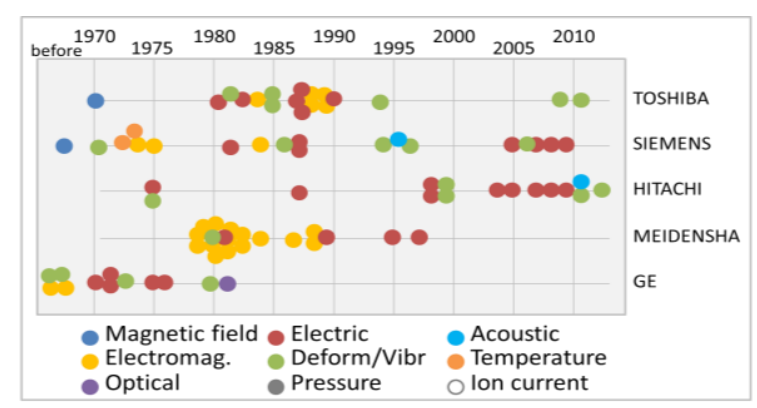

Fig. 3. Performance of pressure sensors - Time distribution of technologies for the TOP 5 players

A different analysis has been provided for compactness. Patents and brochures have been scanned in order to identify the size of different devices proposed in the different circuit breakers size (rating currents: $400 \mathrm{~A}, 600 \mathrm{~A}$, 800A, 1200A, 1600A, 2500A, 3000A, 4000A, $8000 \mathrm{~A}$ and rating voltages: $3,6 \mathrm{kV}, 7,2 \mathrm{kV}, 12 \mathrm{kV}$, $15 \mathrm{kV}, 17,5 \mathrm{kV}, 24 \mathrm{kV}, 27 \mathrm{kV}, 36 \mathrm{kV}, 40 \mathrm{kV})$. Figure 4 suggests results only for $7,2 \mathrm{kV}, 2500 \mathrm{~A}$.

\begin{tabular}{|c|c|c|c|c|c|}
\hline \multicolumn{5}{|c|}{ Gas insulated CB $-7,2 \mathrm{kV}, 2500 \mathrm{~A}$} \\
\hline Electric & Elecrtomag. & Deformat. & Magnet. & Acoustic & Optical \\
\hline $5-10 \mathrm{~cm}^{3}$ & $2-3 \mathrm{~cm}^{3}$ & $8-12 \mathrm{~cm}^{3}$ & $2-3 \mathrm{~cm}^{3}$ & $3-5 \mathrm{~cm}^{3}$ & $6-7 \mathrm{~cm}^{3}$ \\
\hline Ion & Temperat. & Pressure & Piezoelec. & Casimir ef. & Balance \\
\hline $6-7 \mathrm{~cm}^{3}$ & $<1 \mathrm{~cm}^{3}$ & $3-5 \mathrm{~cm}^{3}$ & $<1 \mathrm{~cm} 3$ & $2-4 \mathrm{~cm}^{3}$ & $6-7 \mathrm{~cm}^{3}$ \\
\hline
\end{tabular}

Fig. 4. Compactness of pressure sensors (partial) for version $7,2 k V-2500 A$.

Figures 3 and 4 are just an example, many other graphs and tables have been provided for giving a concise perspective on the position of each technology. Similar analysis have been repeated for all others requirements.

\section{2) Requirements evaluation.}

Results from phase 1 are used by experts to conduct the evaluation process dealing with the market potential of each requirement. An importance-satisfaction map can be generated for the technology used as the reference technology. All others alternative technologies are then mapped in a comparison table, as shown in table 1 , and then quantitatively evaluated by a unique score calculated by a weighted average between satisfaction and market potential. For privacy reasons, values in table 1 are only indicatives.

Table 1 Comparison between pressure sensors technologies, values are modified for privacy reasons

\begin{tabular}{|l|c|c|c|c|c|c|c|}
\hline \multicolumn{1}{|c|}{ Requirements } & $\begin{array}{c}\text { Market } \\
\text { Potent. }\end{array}$ & $\begin{array}{c}\text { Electric } \\
\text { Measur. }\end{array}$ & $\begin{array}{c}\text { Electrom. } \\
\text { Wave }\end{array}$ & $\begin{array}{c}\text { Deform. } \\
\text { Vibration }\end{array}$ & $\begin{array}{c}\text { Magnetic } \\
\text { field }\end{array}$ & $\begin{array}{c}\text { Sonic } \\
\text { waves }\end{array}$ & $\ldots$ \\
\hline 1 Power Consump. & 3,0 & 80,0 & 70,0 & 90,0 & 50,0 & 80,0 & $\ldots$ \\
\hline 2 Cost & 4,5 & 75,0 & 75,0 & 75,0 & 80,0 & 85,0 & $\ldots$ \\
\hline 3 Maintenance & 10,0 & 100,0 & 80,0 & 60,0 & 70,0 & 70,0 & $\ldots$ \\
\hline 4 Compactness & 23,0 & 20,0 & 90,0 & 40,0 & 90,0 & 80,0 & $\ldots$ \\
\hline 5 Sensitivity & 16,0 & 40,0 & 80,0 & 60,0 & 60,0 & 70,0 & $\ldots$ \\
\hline 6 Calibration & 14,0 & 60,0 & 30,0 & 40,0 & 80,0 & 30,0 & $\ldots$ \\
\hline 7 ... & $\ldots$ & $\ldots$ & $\ldots$ & $\ldots$ & $\ldots$ & $\ldots$ & $\ldots$ \\
\hline PRODUCT VALUE & 77,0 & 86,0 & 78,0 & 82,0 & 69,0 & 73,0 & $\ldots$ \\
\hline
\end{tabular}

\section{Conclusions}

"KOMpetitive Intelligence" is a systematic procedure to foster the ability of experts to identify and assess technological alternatives on the base of two parameters, importance and satisfaction. The evaluation is based on quantitative and objective data coming from strategic knowledge extracted from patents, scientific literature, web and problem solving/evolutionary theory. The main limit of the evaluation is to consider requirements as independent from each other to facilitate the judgment of experts. Evaluation is supported by concise and easy diagram (preferably infographics) that allows engineers and decision makers to have a comprehensive and fast overview on the situation, increasing awareness and consistency of decision making. This methodology has already been tested and adopted by multinational corporations.

\section{References}

[1] Patent US8943371 (B2), Methods and systems for computer-aided identification of technical phenomena.

[2] G.S. Altshuller, Creativity as an exact science, Gordon and Breach (1984).

[3] G.S. Altshuller, L. Shulyak, S. Rodman, The innovation algorithm: TRIZ, systematic innovation and technical creativity. Technical Innovation Center, Inc. (1999).

[4] Y. Salamatov, The right solution at the right time, a guide to innovative problem solving, Insytech (1999).

[5] D. Russo, T. Montecchi, Y. Liu, Functional-based search for patent technology transfer. In ASME 2012 International Design Engineering Technical Conferences and Computers and Information in Engineering Conference, 529-539 (2012).

[6] T. Montecchi, D. Russo, Y. Liu, Searching in Cooperative Patent Classification: Comparison between keyword and concept-based search, Advanced Engineering Informatics, 27(3), 335345 (2013).

[7] J. Jacoby, The emerging behavioral process technology in consumer decision-making. In: W. D. Perreault Jr. (Ed.), Advances in consumer research, vol. 4, 263-265 (1977).

[8] P.W. Farris, et al., Marketing metrics: The definitive guide to measuring marketing performance. Pearson Education (2010).

[9] A.W. Ulwick, An Introduction to Outcome-Driven Innovation. Strategyn Inc. (2009). 